\title{
Electron states and electron-phonon coupling in the BEDT-TTF-based organic superconductors
}

\author{
J. Shumway \\ Department of Physics and Astronomy, University of Missouri, Columbia, Missouri 65211 \\ S. Chattopadhyay \\ Department of Physics, Columbia College, Columbia, Missouri 65216 \\ S. Satpathy \\ Department of Physics and Astronomy, University of Missouri, Columbia, Missouri 65211
}

(Received 18 September 1995)

\begin{abstract}
The electronic structure and the coupling of electrons to the vibrational modes of a single bis (ethylenedithio)-tetrathiafulvalene (BEDT-TTF) molecule, the building block of a class of organic superconductors, are studied from quantum chemical calculations. We find that the molecular orbital structure near the Fermi energy can be described with a simple tight-binding model involving only the out-of-plane $p$ orbitals on the carbon and sulfur atoms in the central part of the molecule. Just three of the vibrational modes of $A_{g}$ symmetry couple strongly to the electron states resulting in a frequency shift of typically $10 \%$ for these modes. The high-frequency $A_{g}(11)$ mode is predicted to be broadened the most by the coupling, by an amount of $\sim 8 \%$. The calculated electron-phonon coupling constant in the solid, $\lambda \sim 0.4$, is strong enough to produce a superconducting transition temperature $T_{c} \sim 3-10 \mathrm{~K}$ as estimated from McMillan's formula, indicating that the superconductivity in the BEDT-TTF compounds can be explained within the standard BCS electron-phonon coupling theory.
\end{abstract}

\section{INTRODUCTION}

Since the introduction of the first organic superconductors in the early 1980s by Bechgaard and co-workers, ${ }^{1}$ a number of organic superconductors have been synthesized so far. The more recent sulfur-based cation bis (ethylenedithio)tetrathiafulvalene (BEDT-TTF) (ET, for short ${ }^{2}$ ) has produced a series of quasi-two-dimensional compounds with steadily increasing superconducting transition temperatures. The ET series includes the $\beta$ phase compounds $\beta$-(ET) ${ }_{2} X\left(X^{-}\right.$ $=\mathrm{I}_{3}{ }^{-}, \mathrm{IBr}_{2}{ }^{-}$, etc. $)$, the $\kappa$ phase compounds $\kappa$-(ET) ${ }_{2} X\left(X^{-}\right.$ $=\mathrm{I}_{3}{ }^{-}, \mathrm{Cu}(\mathrm{NCS})_{2}{ }^{-}$, etc.), and the $\theta$ phase compounds such as $\theta-(\mathrm{ET})_{2} \mathrm{I}_{3}$. Of the ten or so superconductors found in the ET series so far, the compound $\kappa-(\mathrm{ET})_{2} \mathrm{Cu}\left[\mathrm{N}(\mathrm{CN})_{2}\right] \mathrm{Br}$ has the highest $T_{c}$ of $11.6 \mathrm{~K}$ at ambient pressure. ${ }^{3}$

The ET superconductors consist of layers of ET molecules and layers of anions alternately stacked. The ET layers are the metallic layers and the anion layers form the insulating layers in a manner somewhat analogous to the high- $T_{c}$ copper-oxide superconductors. In this paper, we analyze the electronic structure of a single ET molecule and examine the strength of the electron-phonon coupling to determine whether it is a plausible mechanism for superconductivity in the ET solids.

\section{ELECTRON STATES}

The structure of the ET molecule, chemical formula of $\mathrm{C}_{10} \mathrm{~S}_{8} \mathrm{H}_{8}$, is sketched in Fig. 1. The molecule is roughly planar except for the hydrogen atoms which are attached to the tetrahedral $\left(s p^{3}\right)$ bonds on the terminal carbons. The individual atom positions vary slightly from solid to solid and the terminal carbons occurring slightly off plane can be in either the so-called " $\mathrm{A}$ " or the " $\mathrm{B}$ " conformation. ${ }^{4}$ However, this slight alteration in the atom positions between solids does not introduce any essential change into the molecular-orbital scheme. In view of this, we have chosen here a symmetric form of the molecule with $D_{2 h}$ symmetry for detailed study.
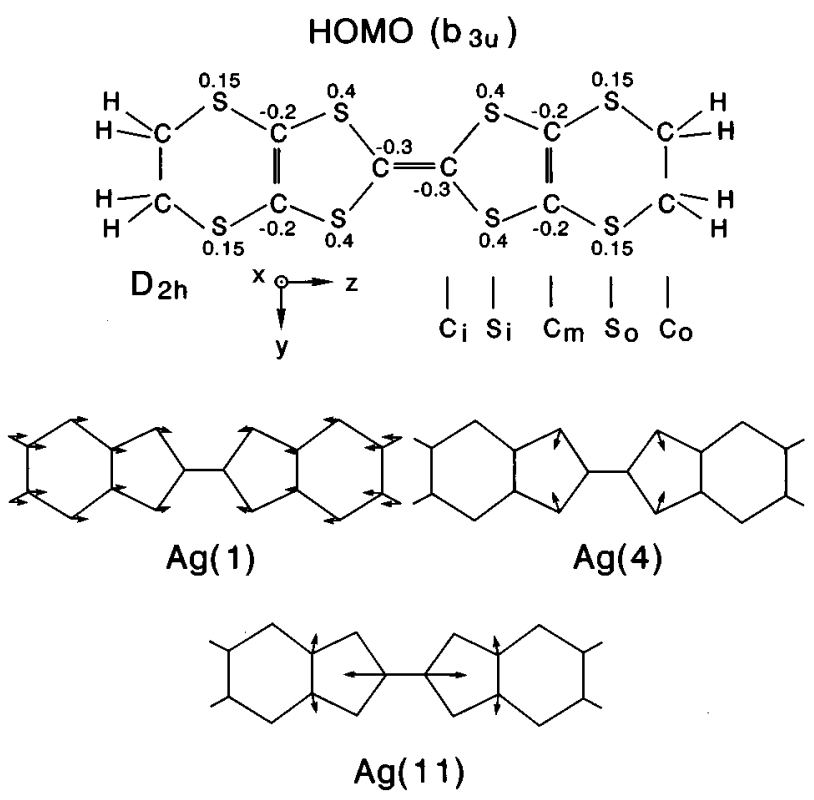

FIG. 1. Sketch of the ET molecule and the calculated HOMO wave function. The amplitude contributions of the $p_{x}$ atomic orbitals to the HOMO wave function are indicated next to each atom. The bottom part shows the three $A_{g}$ vibrational modes that couple strongly to the HOMO. 


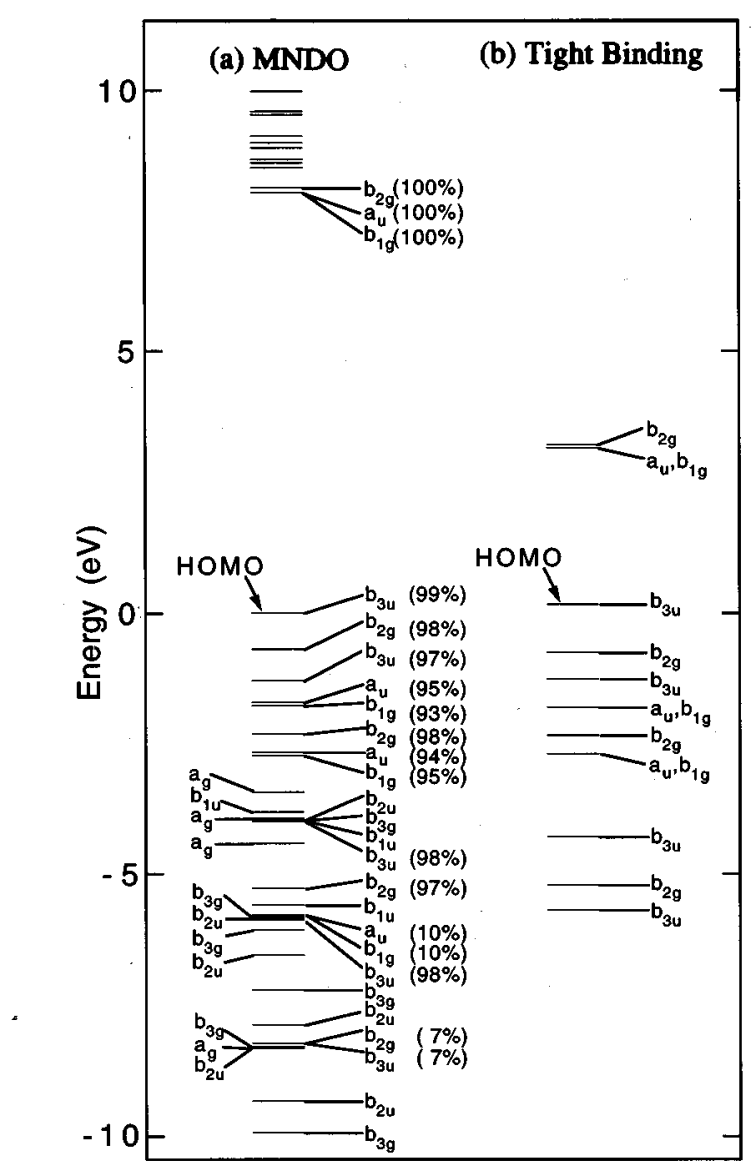

FIG. 2. The calculated MO scheme from the MNDO method (a) and from the model tight-binding Hamiltonian (b). Percentages indicate the contribution of the $p_{x}$ orbitals on the $\mathrm{C}_{i}, \mathrm{C}_{m}, \mathrm{~S}_{i}$, and $\mathrm{S}_{o}$ atoms, taken together, to the MNDO wave functions.

In Fig. 2 we show the molecular-orbital scheme obtained from calculations using the quantum-chemical modified neglect of differential overlap (MNDO) technique..$^{5}$ This technique has been successfully used on a wide variety of compounds and yields reasonable results for molecular orbitals, vibrational frequencies, relative energies, molecular structures, and other physical quantities.

We find that the molecular orbitals (MO's) around the Fermi energy $E_{f}$ are made up of out-of-plane $p_{x}$ orbitals of the carbon and sulfur atoms in the middle part of the molecule, i.e., neither the terminal carbon atoms $\mathrm{C}_{o}$ nor the hydrogen atoms contribute significantly to these MO's. To understand the MO scheme, we note as indicated in Fig. 1 that there are three types of carbon atoms, viz., the outer, inner, and middle carbons denoted by $\mathrm{C}_{o}, \mathrm{C}_{i}$, and $\mathrm{C}_{m}$, respectively, and two types of sulfur atoms (outer and inner) denoted by $\mathrm{S}_{o}$ and $\mathrm{S}_{i}$. With the electronic configurations of $\mathrm{C}=[\mathrm{He}] 2 s^{2} 2 p^{2}, \mathrm{~S}=[\mathrm{Ne}] 3 s^{2} 3 p^{4}$, we have altogether 96 valence electrons for the ET molecule including the hydrogen electrons. It is convenient to think in terms of $s p^{3}$-hybrid orbits for the fourfold-coordinated outer carbon atoms and $s p^{2}$-hybrid orbitals plus $p_{x}$ orbitals for the rest of the carbon atoms, which are threefold coordinated. Each of the four $s p^{3}$-hybrid orbitals on an outer carbon atom interacts strongly with either the hydrogen $s$ orbitals, the in-plane sulfur $p_{y} / p_{z}$ orbitals, or a similar $s p^{3}$ orbital on the neighboring $\mathrm{C}_{o}$ atom. The strong interaction causes the bonding and the antibonding states to be removed away from $E_{f}$ with the result that neither the outer carbon $\left(\mathrm{C}_{o}\right)$ nor the hydrogen atoms have any significant contribution to the MO wave functions near $E_{f}$. Similar strong interactions remove the C$s p^{2}$ orbitals and the remaining sulfur $p_{y}, p_{z}$ orbitals away from $E_{f}$. We are then left with the out-of-plane $p_{x}$ orbitals on the inner and the middle carbon atoms as well as on the sulfur atoms. Now, the sizable $p p \pi$ interaction between the $p_{x}$ orbitals on the adjacent $\mathrm{C}_{i}$ or $\mathrm{C}_{m}$ pairs of atoms causes the $p p \pi^{*}$ states to be shifted up in energy. These in fact form the lowest unoccupied molecular-orbital (LUMO) states (of symmetry $b_{1 g}, b_{2 g}$, and $a_{u}$ ), leaving the $p p \pi$ hybrid orbitals on the $\mathrm{C}_{i}$ or $\mathrm{C}_{m}$ atoms plus the sulfur $p_{x}$ orbitals to form the highest occupied molecular orbitals (HOMO's).

This picture of chemical bonding in the molecule is verified by the results of our MNDO calculations. The symmetries of the calculated MO's are consistent with the above analysis. As seen from Table I, where we have listed the irreducible representations spanned by various orbitals, the $p_{x}$ orbitals on the $\mathrm{C}_{i}, \mathrm{C}_{m}, \mathrm{~S}_{i}$, and $\mathrm{S}_{o}$ atoms together span the $3 b_{1 g}+4 b_{2 g}+3 a_{u}+4 b_{3 u}$ representations of the $D_{2 h}$ group. These orbitals may be identified around the HOMOLUMO gap in the molecular-orbitals scheme. Of these, the three two-centered carbon $p p \pi^{*}$ orbitals span the $b_{1 g}+b_{2 g}$ $+a_{u}$ representations and they form the first three LUMO's as anticipated. The projected density-of-states shown in Fig. 3, where we have broadened the individual MO's by a finiteenergy width for clarity of presentation, confirms again the picture that the $p_{x}$ orbitals on the $\mathrm{S}_{i}, \mathrm{~S}_{o}, \mathrm{C}_{i}$, and $\mathrm{C}_{m}$ atoms form the MO's around the HOMO-LUMO gap. The calculated HOMO-LUMO gap from the MNDO technique is usually too large and we estimate a gap value of about $3.0 \mathrm{eV}$ in the compound based on a tight-binding fit to the energies of the HOMO's as discussed below.

Since the MO's near the HOMO-LUMO gap were found to be made almost exclusively from the out-of-plane $p_{x}$ orbitals on the $\mathrm{S}_{i}, \mathrm{~S}_{o}, \mathrm{C}_{i}$, and $\mathrm{C}_{m}$ atoms, it is useful to obtain a simplified tight-binding Hamiltonian with a minimal basis set of the $14 p_{x}$ orbitals, each located on one of the atoms. Thus we have the simplified, $14 \times 14$ tight-binding Hamiltonian

$$
H=\sum_{i} \varepsilon_{i}|i\rangle\langle i|+\sum_{\langle i, j\rangle} V_{i j}(|i\rangle\langle j|+\text { c.c. }),
$$

where $|i\rangle$ denotes the $p_{x}$ orbital on the $i$ th atom and $\langle i, j\rangle$ denotes nearest-neighbor pairs. By fitting to the energies of the appropriate HOMO's obtained from the MNDO calculation, the parameters of the Hamiltonian (1) were determined to be $\varepsilon_{\mathrm{C}}=-0.9 \mathrm{eV}, \varepsilon_{\mathrm{S}}=-1.9 \mathrm{eV}, V_{\mathrm{C}-\mathrm{C}}=-3.0 \mathrm{eV}$, and $V_{\mathrm{C}-\mathrm{S}}=-1.5 \mathrm{eV}$.

This simplified model introduces an accidental degeneracy in the energy spectrum between the MO's of $b_{1 g}$ and $a_{u}$ symmetries. The accidental degeneracy arises because (i) a function belonging to $b_{1 g}$ can always be constructed from an $a_{u}$ function by switching its sign on one side of the molecule and (ii) the inner carbon atoms $\mathrm{C}_{i}$ do not contribute to the MO's of these symmetries. Even though this accidental degeneracy is strictly true only in the nearest-neighbor 
TABLE I. Irreducible representations of the $D_{2 h}$ group spanned by various sets of orbitals in the ET molecule (Ref. 6). $N$ is the total number of orbitals in the molecule or the number of normal modes. $A$ denotes any of the atoms $\mathrm{S}_{i}, \mathrm{C}_{m}, \mathrm{~S}_{o}$, or $\mathrm{C}_{o}$.

\begin{tabular}{|c|c|c|c|c|c|c|c|c|c|}
\hline & \multirow[b]{2}{*}{$N$} & \multicolumn{8}{|c|}{ Irreducible representations } \\
\hline & & $a_{g}$ & $b_{1 g}$ & $b_{2 g}$ & $b_{3 g}$ & $a_{u}$ & $b_{1 u}$ & $b_{2 u}$ & $b_{3 u}$ \\
\hline \multicolumn{10}{|l|}{ Atomic orbitals: } \\
\hline $\mathrm{C}_{i}-s$ or $\mathrm{Ci}-p_{z}$ & 2 & 1 & & & & & 1 & & \\
\hline $\mathrm{C}_{i}-p_{x}$ & 2 & & & 1 & & & & & 1 \\
\hline $\mathrm{C}_{i}-p_{y}$ & 2 & & & & 1 & & & 1 & \\
\hline$A-s$ or $A-p_{y}$ or $A-p_{z}$ & 4 & 1 & & & 1 & & 1 & 1 & \\
\hline$A-p_{x}$ & 4 & & 1 & 1 & & 1 & & & 1 \\
\hline H- $s$ or $p_{x}$ or $p_{y}$ or $p_{z}$ & 8 & 1 & 1 & 1 & 1 & 1 & 1 & 1 & 1 \\
\hline $\mathrm{C}_{i}-p p \pi$ & 1 & & & & & & & & 1 \\
\hline $\mathrm{C}_{i}-p p \pi^{*}$ & 1 & & & 1 & & & & & \\
\hline $\mathrm{C}_{m}-p p \pi$ & 2 & & & 1 & & & & & 1 \\
\hline $\mathrm{C}_{m}-p p \pi^{*}$ & 2 & & 1 & & & 1 & & & \\
\hline $\mathrm{C}_{i}, \mathrm{C}_{m}, \mathrm{~S}_{i}, \mathrm{~S}_{o}-p_{x}$ & 14 & & 3 & 4 & & 3 & & & 4 \\
\hline Normal modes: & 72 & 12 & 6 & 7 & 11 & 7 & 11 & 11 & 7 \\
\hline \multicolumn{10}{|c|}{ Raman active: $a_{g}, b_{1 g}, b_{2 g}, b_{3 g} ;$ IR active: $b_{1 u}, b_{2 u}, b_{3 u}$} \\
\hline \multicolumn{10}{|c|}{ Dipole-allowed electronic transitions: } \\
\hline$x$-polarized light: & $a_{g} \leftrightarrow b_{3 u}$ & \multirow{3}{*}{\multicolumn{2}{|c|}{$\begin{array}{c}b_{1 g} \leftrightarrow b_{2 u} \\
b_{1 g} \leftrightarrow b_{3 u} \\
b_{1 g} \leftrightarrow a_{u}\end{array}$}} & \multirow{3}{*}{\multicolumn{2}{|c|}{$\begin{array}{c}b_{2 g} \leftrightarrow b_{1 u} \\
b_{2 g} \leftrightarrow a_{u} \\
b_{2 g} \leftrightarrow b_{3 u}\end{array}$}} & \multirow{3}{*}{\multicolumn{2}{|c|}{$\begin{aligned} b_{3 g} \leftrightarrow a_{u} \\
b_{3 g} \leftrightarrow b_{1 u} \\
b_{3 g} \leftrightarrow b_{2 u}\end{aligned}$}} & & \\
\hline$y$-polarized light: & $a_{g} \leftrightarrow b_{2 u}$ & & & & & & & & \\
\hline z-polarized light: & $a_{g} \leftrightarrow b_{1 u}$ & & & & & & & & \\
\hline $\begin{array}{l}\text { Electron-phonon couplin } \\
(\Gamma \times \Gamma)_{\text {sym }}=A_{g} \text { for all } \Gamma\end{array}$ & & & & & & & & & \\
\hline
\end{tabular}

model, it should be noted that the $b_{1 g}$ and $a_{u}$ MO's are still found in pairs in the MNDO results, indicating the smallness of the next-neighbor interactions, providing an additional justification for retaining only the nearest-neighbor interaction in the Hamiltonian (1). The energies of the LUMO and LUMO +1 are very close because of this effect.

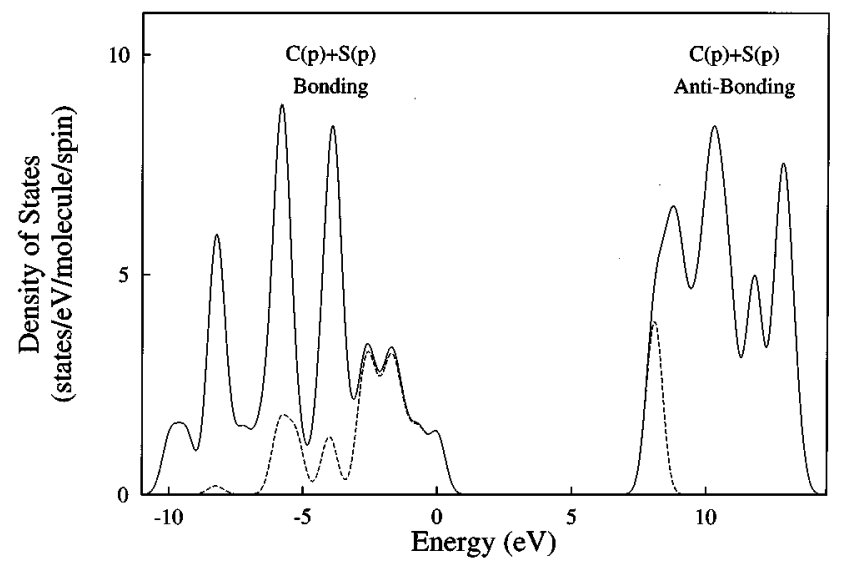

FIG. 3. Densities of states (DOS) around the HOMO-LUMO gap as calculated from the MNDO method: (1) total DOS (solid line) and (2) partial DOS corresponding to the $p_{x}$ orbitals on the $\mathrm{C}_{i}, \mathrm{C}_{m}, \mathrm{~S}_{i}$, and $\mathrm{S}_{o}$ atoms (dashed line). The MO levels have been broadened by about $0.3 \mathrm{eV}$. The predominant contribution of these $p_{x}$ orbitals to the MO's around the HOMO-LUMO gap leads to the model Hamiltonian, Eq. (1), as discussed in the text.
The wave function of the HOMO, of $b_{3 u}$ symmetry, has been illustrated in Fig. 1. The wave function has $68 \%$ of S- $p_{x}$ and $32 \%$ of C- $p_{x}$ character and is consistent with the scanning tunneling microscopy data obtained for the $\kappa-(\mathrm{ET})_{2} \mathrm{Cu}(\mathrm{NCS})_{2}$ compound, which indicated a large concentration of the electronic charge on the carbon and the sulfur atoms in the central part of the molecule. ${ }^{7}$ The HOMO is separated from the HOMO-1, of $b_{2 g}$ symmetry, by about $0.9 \mathrm{eV}$ in our calculation. The HOMO broadens out to form the conduction bands in the solid, estimated to be about 1.0$1.2 \mathrm{eV}$ wide, ${ }^{8}$ such small widths being typical of molecular solids. The $b_{3 u}$ band, which may sometimes be split in the solid due to interaction between two neighboring ET molecules, is partially depleted because of the electron transfer to the anions, and as a result this is the band that occurs at $E_{f}$.

\section{ELECTRON-PHONON COUPLING}

The question we address now concerns the plausibility of the electron-phonon ( $e$-ph) coupling as a mechanism for superconductivity in the ET solids. A phonon affects the electronic energies by altering the electronic hopping matrix elements caused by the change in the interatomic distances. The basic quantity describing this interaction is the $e$-ph matrix element ${ }^{9,10}$

$$
g_{\nu, n k, m(k+q)}=\left\langle n k\left|\sum_{i} e_{i \nu q} \sqrt{\hbar /\left(2 M_{i} \omega_{\nu q}\right)} \frac{\partial H}{\partial R_{i}}\right| m, k+q\right\rangle,
$$


TABLE II. Electron-phonon coupling of the HOMO to the vibrational modes of the ET molecule. $P$ denotes the percentage contribution from the $\mathrm{C}_{i}, \mathrm{C}_{m}, \mathrm{~S}_{i}$, and $\mathrm{S}_{o}$ atoms to the normal mode.

\begin{tabular}{lcccccccccccc}
\hline \hline Phonon & $A_{g}(1)$ & $A_{g}(2)$ & $A_{g}(3)$ & $A_{g}(4)$ & $A_{g}(5)$ & $A_{g}(6)$ & $A_{g}(7)$ & $A_{g}(8)$ & $A_{g}(9)$ & $A_{g}(10)$ & $A_{g}(11)$ & $A_{g}(12)$ \\
\hline$\omega_{\text {exp }}\left(\mathrm{cm}^{-1}\right)^{\mathrm{a}}$ & 165 & 316 & 467 & 502 & 649 & 904 & 980 & 1274 & 1407 & 1474 & 1511 & 2968 \\
$\omega_{\text {calc }}\left(\mathrm{cm}^{-1}\right)$ & 176 & 354 & 583 & 606 & 816 & 1174 & 1282 & 1362 & 1443 & 1737 & 1797 & 3227 \\
$P(\%)$ & 35 & 65 & 72 & 100 & 8.1 & 87 & 0.4 & 0.1 & 0.0 & 99 & 100 & 0.0 \\
$\gamma_{\nu} / \omega_{\nu}(\%)$ & 0.5 & 0.1 & 0.1 & 2.5 & 0.0 & 0.0 & 0.0 & 0.0 & 0.0 & 0.3 & 8.1 & 0.0 \\
$\Delta \omega_{\nu} / \omega_{\nu}(\%)$ & 9.4 & 1.1 & 0.7 & 12.5 & 0.1 & 0.0 & 0.0 & 0.0 & 0.0 & 0.5 & 13.8 & 0.0 \\
$g_{\nu}(\mathrm{meV})$ & 35 & 17 & 17 & 75 & 8 & 6 & 1 & 2 & 0 & 25 & 136 & 0 \\
$V_{\nu}(\mathrm{meV})$ & 112 & 13 & 8 & 150 & 1 & 0.5 & 0 & 0 & 0 & 6 & 166 & 0 \\
\hline \hline
\end{tabular}

${ }^{\mathrm{a}}$ Reference 11 .

where $|m k\rangle$ is a band electron state with the band index $m$ and Bloch momentum $k, e_{i \nu q}$ is the phonon eigenvector with frequency $\omega_{\nu q}$, and the index $i$ runs over all atoms as well as the Cartesian coordinates. The dimensionless $e$-ph coupling constant $\lambda$ is then given by a double integration over the Fermi surface

$$
\begin{aligned}
\lambda= & \sum_{\nu q} \frac{2}{\hbar \omega_{\nu q} N(0)} \sum_{n m k}\left|g_{\nu, n k, m(k+q)}\right|^{2} \\
& \times \delta\left(\varepsilon_{n k}\right) \delta\left(\varepsilon_{m, k+q}-\varepsilon_{n k}-\hbar \omega_{\nu q}\right) .
\end{aligned}
$$

Here $N(0)$ is the electronic density of states at $E_{f}$ per spin and the energies are measured with respect to $E_{f}$. Since we are concerned only with the partially filled HOMO band, of $b_{3 u}$ symmetry, the $n, m$ summation in (3) over the bands is reduced to summing over just this band. If we now neglect the weak intermolecular coupling as compared to the strong intramolecular coupling, the double integration can be performed analytically to yield

$$
\lambda=N(0) V_{e-\mathrm{ph}},
$$

where

$$
V_{e-\mathrm{ph}}=\sum_{\nu} V_{\nu}, \text { with } V_{\nu}=\frac{2}{\hbar \omega_{\nu}}\left|g_{\nu}\right|^{2},
$$

so that the strength of the $e$-ph coupling in the solid can be obtained by computing how the vibrational normal modes are coupled to the electron states of the isolated molecule. The quantity $g_{\nu}$ in (4) is then determined by how a vibrational mode in the isolated molecule affects the energy of the HOMO, i.e.,

$$
g_{\nu}=\left\langle\operatorname{HOMO}\left|\sum_{i} e_{i \nu} \sqrt{\hbar /\left(2 M_{i} \omega\right)} \frac{\partial H}{\partial R_{i}}\right| \text { HOMO }\right\rangle .
$$

The $e$-ph coupling produces a softening of the phonon frequency in the solid given by $\Delta \omega_{\nu}=-2 N(0)\left|g_{\nu}\right|^{2}$ and a phonon linewidth of $\gamma_{\nu}=2 \pi N(0)^{2} \omega_{\nu}\left|g_{\nu}\right|^{2}$.

Not all vibrational modes couple to the electron states in the molecule. Group theory tells us that the coupling is nonzero only if $\left(\Gamma_{\mathrm{el}} \times \Gamma_{\mathrm{el}}\right)_{\mathrm{sym}}=\Gamma_{\mathrm{vib}}$. As indicated in Table I, this means that only the $A_{g}$ vibrational modes can couple to the electron states for the $D_{2 h}$ group. There are in total $12 A_{g}$ vibrational modes and now we proceed to compute the strength of the $e$-ph coupling for these modes.
The computation of the $e$-ph coupling strength requires theoretical models for (a) the vibrational normal modes, (b) the electron states of the molecule, and (c) the way in which the electron states change in response to the atomic displacements caused by the vibrational normal modes. The vibrational modes were obtained from the quantum-chemical MNDO calculations. The agreement between the calculated and the measured vibrational frequency is reasonable (Table II), especially in view of the fact that the calculations were performed on the symmetric molecule. The electron states are described here by the tight-binding Hamiltonian, Eq. (1), involving just the minimal basis set of the $14 p_{x}$ orbitals. This is reasonable since as seen from Fig. 2 these $p_{x}$ orbitals contribute about $99 \%$ of the wave function of the HOMO. We finally need a model for how the electron states change in response to the change in distance between atoms. This we incorporate by simply scaling the hopping integrals in Eq. (1) with distance $d$ between nearest-neighbor atoms, following Harrison's universal formula ${ }^{12}$

$$
V_{l l^{\prime} m}=\eta_{l l^{\prime} m} \frac{\hbar^{2}}{m d^{\eta}},
$$

with the exponent $\eta=2$ for the $p$ - $p$ matrix elements.

The $e$-ph couplings calculated from Eqs. (2)-(5) are shown in Table II. As seen from the table, only three of the $12 A_{g}$ vibrational modes, the $A_{g}(1), A_{g}(4)$, and $A_{g}(11)$ modes, with the calculated frequencies of 176,606 , and 1797 $\mathrm{cm}^{-1}$, respectively, couple strongly to the HOMO. Indeed a strong coupling for the 502 and $1474 \mathrm{~cm}^{-1}$ modes are found in the experiments of Sugai et al. ${ }^{13}$ (The coupling for the lower $176 \mathrm{~cm}^{-1}$ mode was not reported in the experiments.) The $A_{g}(11)$ mode for our symmetric molecule presumably corresponds to the experimental $1474 \mathrm{~cm}^{-1}$ mode. ${ }^{14}$ The coupling for this vibrational mode is strong because of the simultaneous stretching of all $\mathrm{C}=\mathrm{C}$ bonds (see Fig. 1) on which a large part of the HOMO wave function is located. In contrast, the nearby $A_{g}(10)$ mode with $\omega_{\text {calc }}=1737 \mathrm{~cm}^{-1}$ has a rather small coupling because while the central $\mathrm{C}=\mathrm{C}$ bond is stretched, the other two are compressed, leading to a cancellation effect. The $e$-ph coupling results in a softening of the phonon frequency by $\sim 10 \%$ for these three modes and a relatively large linewidth for the two higher modes as indicated in the table.

The superconducting transition temperature may be estimated from McMillan's formula, 


$$
T_{c}=\frac{\left\langle\omega_{\log }\right\rangle}{1.2} \exp \left[\frac{-1.04(1+\lambda)}{\lambda-\mu^{*}(1+0.62 \lambda)}\right],
$$

which holds if the lattice is not coupled too strongly to the electrons $(\lambda \leqslant 1.5)$. To estimate a value for $\lambda$, we have used the value for the one-electron density of states $N(0)$ $\sim 0.8-1.0$ states/(eV spin molecule) assuming a bandwidth of $\sim 1.0-1.2 \mathrm{eV}$ for the HOMO band ${ }^{8}$ and a uniform density of states over this energy range. With the calculated $V_{e \text {-ph }}$ $=457 \mathrm{meV}$ (Table II), we then obtain a $\lambda$ value of $0.38-$ 0.45 . With an effective Coulomb pseudopotential parameter of $\mu^{*} \sim 0.1$, McMillan's equation (7) then yields an estimated $T_{c}$ in the range of $3-10 \mathrm{~K}$ in agreement with the typical experimental values of $T_{c}$ for these compounds.

\section{SUMMARY}

In summary, we have examined the nature of electron states in a single ET molecule and have obtained a simple tight-binding model for the molecular orbitals around the Fermi energy. These molecular orbitals arise out of the car- bon and sulfur out-of-plane $p_{x}$ orbitals, while the outer carbon atoms and the hydrogen atoms do not contribute to the states near the gap. This implies in turn that in the solid, where the ET molecules are stacked together to form conducting planes separated by insulating anion planes, the superconducting electrons are confined to the central part of the molecule. Thus the strongest electron-phonon couplings result for those phonon modes with a strong amplitude in the central part of the molecule. We find, in general agreement with the experiment, that just three of the $A_{g}$ phonons couple strongly to the electrons. The estimated value of the $e$-ph coupling parameter, $\lambda \sim 0.4$, is consistent with the BCS electron-phonon mechanism for superconductivity in the ET solids.

\section{ACKNOWLEDGMENTS}

We thank David L. Cowan for stimulating discussions and Rainer Glaser and Christopher Horan for help with numerical computations. Financial support was in part provided by the Research Board of the University of Missouri.
${ }^{1}$ D. Jérome, A. Mazaud, M. Ribault, and K. Bechgaard, J. Phys. Lett. 41, L95 (1980).

${ }^{2}$ BEDT-TTF or ET stands for the cation, bis (ethylenedithio)tetrathiafulvalene, with the chemical formula $\mathrm{C}_{10} \mathrm{H}_{8} \mathrm{~S}_{8}$.

${ }^{3}$ A. M. Kini, U. Geiser, and H. H. Wang, Inorg. Chem. 29, 2555 (1990).

${ }^{4}$ P. C. Leung, T. J. Emge, and M. A. Beno, J. Am. Chem. Soc. 107, 6184 (1985).

${ }^{5}$ M. J. S. Dewar and W. Thiel, J. Am. Chem. Soc. 99, 4899 (1977); 99, 4907 (1977); J. J. P. Stewart, J. Comp. Chem. 10, 221 (1989).

${ }^{6}$ Notations for irreducible representations are from M. Tinkham, Group Theory and Quantum Mechanics (McGraw-Hill, New York, 1964).

${ }^{7}$ M. Yoshimura, H. Shigekawa, H. Nejoh, G. Saito, Y. Saito, and A. Kawazu, Phys. Rev. B 43, 13590 (1991).
${ }^{8}$ M. H. Whangbo, J. M. Williams, and P. C. W. Leung, J. Am. Chem. Soc. 107, 5815 (1985).

${ }^{9}$ C. O. Rodriguez, A. I. Liechtenstein, I. I. Mazin, O. Jepsen, and O. K. Andersen, Phys. Rev. B 42, 2692 (1990).

${ }^{10}$ D. Rainer, Prog. Low Temp. Phys. 10, 371 (1986).

${ }^{11}$ Experimental frequencies are for $\kappa$-(ET) ${ }_{2} \mathrm{Cu}(\mathrm{NCS})_{2}$ (Ref. 13).

${ }^{12}$ W. A. Harrison, Electronic Structure and the Properties of Solids: The Physics of the Chemical Bond (Freeman, San Francisco, 1979).

${ }^{13}$ S. Sugai, H. Mori, Y. Yamochi, and G. Saito, Phys. Rev. B 47, 14374 (1993).

${ }^{14}$ In fact, the MNDO calculation for the real, nonsymmetric molecule yields a frequency of $1427 \mathrm{~cm}^{-1}$ for this vibrational mode in reasonable agreement with the experiment: M. E. Kozlov, K. I. Pokhodnia, and A. A. Yurchenko, Spectrochim. Acta 45A, 437 (1989). 